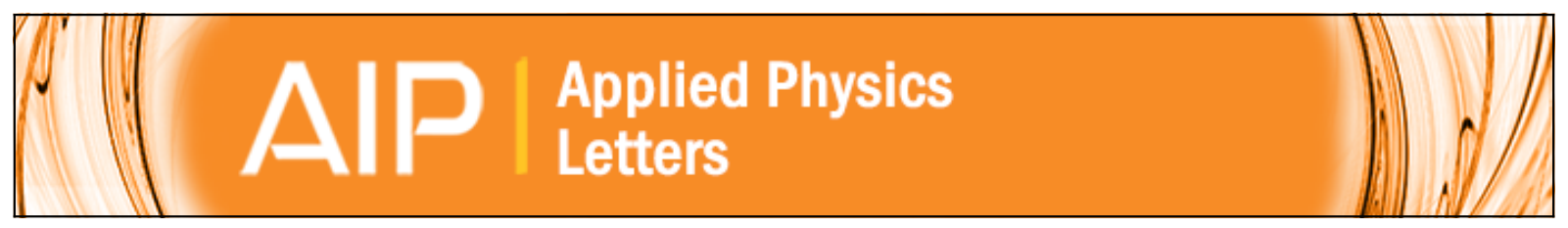

\title{
Large negative persistent photoconductivity in InAs/AISb quantum wells
}

Yu. G. Sadofyev, A. Ramamoorthy, J. P. Bird, S. R. Johnson, and Y.-H. Zhang

Citation: Applied Physics Letters 86, 192109 (2005); doi: 10.1063/1.1926407

View online: http://dx.doi.org/10.1063/1.1926407

View Table of Contents: http://scitation.aip.org/content/aip/journal/apl/86/19?ver=pdfcov

Published by the AIP Publishing

\section{Articles you may be interested in}

Electron spin relaxation time in (110) InGaAs/InAIAs quantum wells

J. Appl. Phys. 116, 023507 (2014); 10.1063/1.4887803

Excitation wavelength dependence of the anomalous circular photogalvanic effect in undoped InGaAs/AIGaAs quantum wells

J. Appl. Phys. 115, 083509 (2014); 10.1063/1.4867039

Relaxation and dephasing of the intersubband transitions in $\mathrm{n}$-type In As / Al Sb multi quantum wells Appl. Phys. Lett. 91, 071918 (2007); 10.1063/1.2769948

Study of Spin-Orbit Interaction in AlxGa1-xAsySb1-y/InAs Quantum Well by Means of Persistent Photoconductivity Effect on Weak Localization

AIP Conf. Proc. 893, 569 (2007); 10.1063/1.2730019

Positive and negative persistent photoconductivities in semimetallic Al x Ga 1-x Sb/lnAs quantum wells J. Appl. Phys. 86, 3152 (1999); 10.1063/1.371182 


\title{
Large negative persistent photoconductivity in InAs/AISb quantum wells
}

\author{
Yu. G. Sadofyev, A. Ramamoorthy, J. P. Bird, S. R. Johnson, a) and Y.-H. Zhang \\ Department of Electrical Engineering and Center for Solid State Electronics Research, Arizona State \\ University, Tempe, Arizona 85287-5706
}

(Received 20 December 2004; accepted 28 March 2005; published online 5 May 2005)

\begin{abstract}
Negative persistent photoconductivity in backgated InAs/AlSb quantum wells under red and blue light emitting diode illumination is observed to depend on both illumination energy and intensity. During these experiments the electron sheet density is varied by one order of magnitude from $5.6 \times 10^{11}$ to $5.8 \times 10^{10} \mathrm{~cm}^{-2}$. This behavior is attributed to the role of optically excited holes in the $\mathrm{AlSb}$ barrier layers near the InAs quantum well. Furthermore, a long relaxation time in the electron sheet density is observed and attributed to the slow movement of electrons from the InAs quantum well to ionized deep levels in the AlSb barriers. (C) 2005 American Institute of Physics. [DOI: $10.1063 / 1.1926407]$
\end{abstract}

InAs/AlSb-based quantum structures are very attractive for applications in spintronics and high-speed electronic devices. The advantages of this material system include the low electron effective mass and large $g^{*}$ factor of InAs and the strong electron confinement that results from the large energy difference $(1.35 \mathrm{eV})$ between the conduction bands of AlSb and InAs. It is well established that, in nominally undoped InAs/AlSb structures, a two-dimensional electron gas (2DEG) is formed in the InAs well with a sheet density on the of order $10^{12} \mathrm{~cm}^{-2}$. The proposed sources of these carriers include donorlike defects in the AlSb barriers, donors at the InAs/AlSb interfaces, and surface donors in the GaSb cap layer. ${ }^{1} \mathrm{An}$ interesting feature of the InAs/AlSb system is a bipolar behavior of its persistent photoconductivity. ${ }^{2}$ Positive persistent photoconductivity, namely, an increase in the 2DEG density, occurs for illumination by photons with energies lower than about $1.55 \mathrm{eV}$, and has been attributed to the photoionization of deep donors. ${ }^{2}$ Negative persistent photoconductivity (NPPC), on the other hand, occurs for illumination by photons with energies larger than about $1.55 \mathrm{eV}$. The NPPC effect has been attributed to photoexcited holes that drift toward, and recombine with electrons in the InAs quantum well (QW) $[2,3]$ and the capture of photoexcited electrons by deep donors in the AlSb barriers that are located slightly above the first quantized subband in the InAs QW. ${ }^{4}$ In similar InAs/AlGaSb structures, which have a larger type-II valance band offset than InAs/AlSb, the NPPC effect has been attributed to the capture of photoexcited electrons from the InAs well by ionized deep donors in the AlGaSb barriers ${ }^{5}$ and compositional fluctuations at the $\mathrm{AlGaSb} / \mathrm{InAs}$ interface. $^{6}$

This article reports the study of the NPPC effect in backgated InAs/AlSb QWs where the electron density in the QW is decreased by a factor of 2.6 using red light emitting diode (LED) illumination and a factor of 9.7 (from $5.6 \times 10^{11}$ to $5.8 \times 10^{10} \mathrm{~cm}^{-2}$ ) using blue light emitting diode (LED) illumination. Previous work reports a factor of two using a red LED (Ref. 7) and factors of three $e^{3}$ and five $e^{7}$ using a green LED which is attributed to the role of photogenerated holes in the AlSb barriers.

\footnotetext{
a) Author to whom correspondence should be addressed; electronic mail:
} shane.johnson@asu.edu
Undoped InAs/AlSb QWs structures were grown on (100) $n^{+}$GaAs substrates by molecular-beam epitaxy. In order to accommodate the lattice-constant differences between $\mathrm{GaAs}, \mathrm{AlSb}$, and InAs, and to increase the electrical resistance between the InAs QW and the GaAs substrate, a composite buffer layer was grown. This layer consisted of 500 $\mathrm{nm}$ of AlAs, grown on a $200 \mathrm{~nm}$ undoped GaAs buffer, followed by a $2 \mu \mathrm{m}$ thick AlSb metamorphic buffer layer, and a 25 -period $(2.5 \mathrm{~nm} / 1.25 \mathrm{~nm}) \mathrm{AlSb} / \mathrm{GaSb}$ superlattice. The superlattice serves as a smoothing layer for subsequent molecular-beam epitaxy growth and as an etch-stop layer during Hall-bar preparation by wet chemical etching. The active part of the structure consists of a $25 \mathrm{~nm}$ AlSb barrier, a $15 \mathrm{~nm}$ InAs QW, a $40 \mathrm{~nm}$ AlSb barrier, and a $6 \mathrm{~nm} \mathrm{GaSb}$ cap layer. These layers were grown at a substrate temperature of $480^{\circ} \mathrm{C}$, and the shutter sequence was chosen to provide InSb-like bonds at both interfaces of the InAs QW.

After growth, Hall bars were defined by mesa etching in a solution of $\mathrm{H}_{3} \mathrm{PO}_{4}: \mathrm{H}_{2} \mathrm{O}_{2}: \mathrm{H}_{2} \mathrm{O}(1: 1: 10)$ at room temperature. Since the developers commonly used in photolithography attack GaSb and AlSb, $10 \mathrm{~nm}$ of amorphous Ge was evaporated on top of the GaSb layer to serve as a protection layer. At low temperatures, where our electricalcharacterization studies were performed, we have confirmed that the Ge layer does not create any significant shunting of the QW layer. The phosphoric acid-based solution removes the Ge film, together with the other layers of the structure. After mesa etching, $\mathrm{Si}_{3} \mathrm{~N}_{4}$ was deposited at a temperature of $50{ }^{\circ} \mathrm{C}$ on the sidewalls and floor of the mesa by plasmaassisted chemical vapor deposition. Several non-alloyed ohmic contacts were formed to the top surface of the InAs QW using a two-step etch in phosphoric acid and ammonia hydroxide based solutions to remove the upper layers of Ge, $\mathrm{GaSb}$, and $\mathrm{AlSb}$, and then directly depositing $\mathrm{Cr}-\mathrm{Au}$ on the exposed InAs. Last, an In film was deposited on a backside of the $n^{+}$GaAs wafer to serve as a backgate.

In our study of NPPC, red and blue LEDs were mounted in close proximity to the Hall-bar sample and a predetermined constant current $I_{\text {LED }}$ was supplied to either the blue or the red LED, for an interval of a about $30 \mathrm{~s}$. The influence of the illumination was monitored by measuring the change in the voltage drop between pairs of potential probes, both in the absence and in the presence of a small applied magnetic field. After each dose of illumination, relaxation effects were 


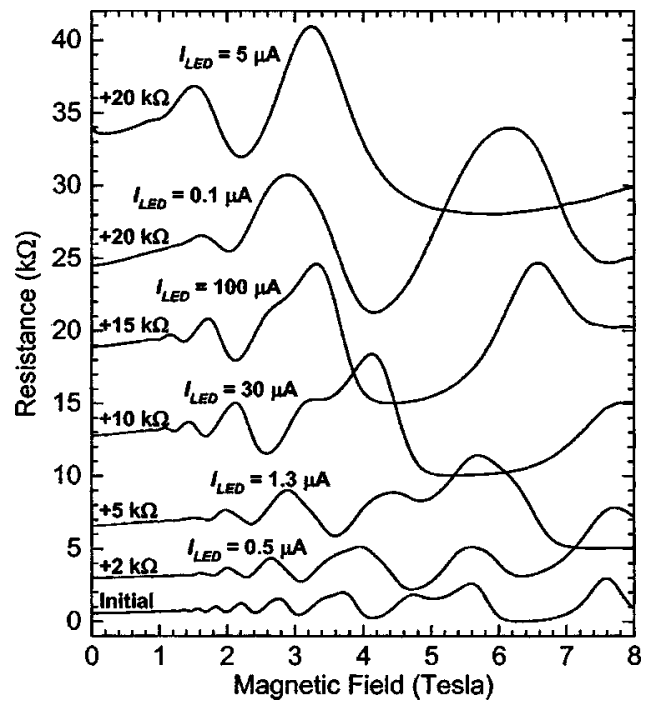

FIG. 1. Shubnikov-de Haas oscillations measured before (lower curve) and after illumination with red light (next four curves) and blue light (upper two curves). For clarity, the curves are shifted vertically by the resistances shown on the left.

observed in the resistance; therefore we waited for approximately $30 \mathrm{~min}$, before beginning the magnetotransport measurements.

The influence of illumination from the red and blue LEDs on the longitudinal magneto-resistance and the 2DEG sheet density is shown in Fig. 1 using zero backgate voltage $\left(V_{g}=0\right)$. The lower curve shows the Shubnikov-de Haas oscillations for the initial measurements performed at $4.2 \mathrm{~K}$, where the electron sheet density is $5.6 \times 10^{11} \mathrm{~cm}^{-2}$ and the electron mobility is $98950 \mathrm{~cm}^{2} / \mathrm{Vs}$. (At $300 \mathrm{~K}$ the electron sheet density was $8.1 \times 10^{11} \mathrm{~cm}^{-2}$ and the electron mobility was $28730 \mathrm{~cm}^{2} / \mathrm{Vs}$.) The next four curves show measurements performed after illumination from the red LED, where the 2DEG density is reduced to $2.1 \times 10^{11} \mathrm{~cm}^{-2}$ for the highest level of illumination used $\left(I_{\mathrm{LED}}=100 \mu \mathrm{A}\right)$. The upper two curves show the measurements performed after illumination from the blue LED, where a further decrease in the electron density is realized. Although not shown for clarity, a 2DEG density of $5.8 \times 10^{10} \mathrm{~cm}^{-2}$ is reached for $I_{\mathrm{LED}}=50 \mu \mathrm{A}$. At this density, the extreme quantum limit is reached, where only the lowest spin-resolved Landau level is occupied at a magnetic field as low as $3 \mathrm{~T}$. The illumination effects are cumulative, starting with the lowest to the highest red LED drive current and then from the lowest to the highest blue LED drive current. Upon warmup and recooling and repeating a blue LED measurement we find that the 2DEG density is reproduced within $20 \%$, indicating that the cumulative effect does not substantially impact the results.

The presence of the composite buffer layer in this structure permits the application of backgate voltages in the range $-7.5<V_{g}<+7.5 \mathrm{~V}$, with a leakage current below $\pm 10 \mathrm{nA}$. After each red LED illumination step, the magnetoresistance was measured for different backgate voltages $\left(V_{g}=0,-0.5\right.$, and $+1.5 \mathrm{~V}$ ). In general, the influence of the backgate was found to be similar to that observed prior to illumination, with a negative (positive) backgate voltage reducing (increasing) the 2DEG sheet density. The low temperature 2DEG mobility decreases linearly with sheet density (see Fig. 2); due to a decrease in electron screening of impurity scatters.

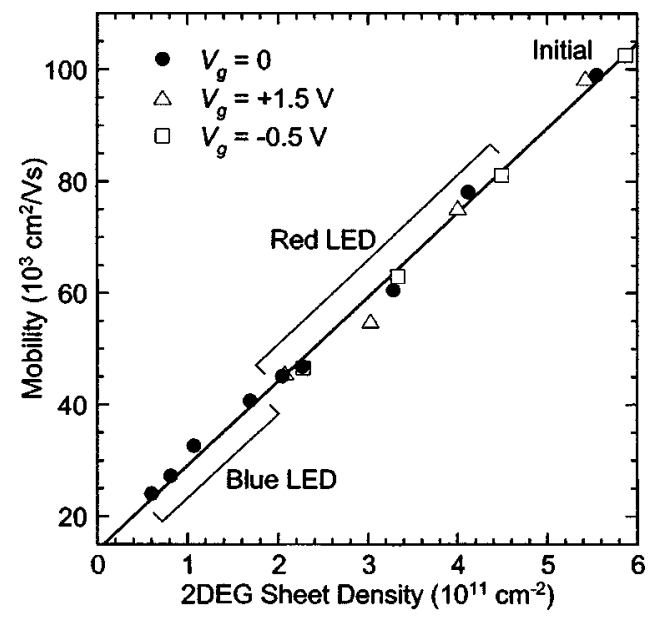

FIG. 2. Linear increase in electron mobility with sheet density under different backgate voltages $\left(V_{g}=0,-0.5\right.$, and $\left.+1.5 \mathrm{~V}\right)$, before (labeled initial) and after illumination with red light (labeled red LED) and blue light (labeled blue LED).

The resistance relaxation in our sample is shown in Fig. 3 , where $30 \mathrm{~s}$ of illumination under the blue LED at a $1 \mu \mathrm{A}$ drive current, increases the resistance well above the initial steady-state value, then after turning the LED off the resistance approaches a new higher resistance. A similar type of behavior is observed using the red LED, although the effect of red light on the sample resistance is not as dramatic. As is consistent with the NPPC effect, this new steady state is a metastable one, where after thermal cycling between room temperature and $4.2 \mathrm{~K}$ the electron concentration and the resistance of the sample return to their initial values. The inset in Fig. 3 shows a long time-scale relaxation process that takes place after illumination where the resistance $R$ increases a further $4 \mathrm{k} \Omega$, saturating at $R_{\mathrm{SAT}}=17 \mathrm{k} \Omega$. This long scale relaxation involves a single time constant of $7740 \mathrm{~s}$ and is likely a result of the slow movement of electrons from the $\mathrm{QW}$ to ionized deep levels in the AlSb barriers. Interface states, or fluctuations of the potential at the InAs QW interfaces, can be ruled out since a random distribution of energies is typical for defect states at interfaces, and should not give rise to a relaxation process with a single time constant.

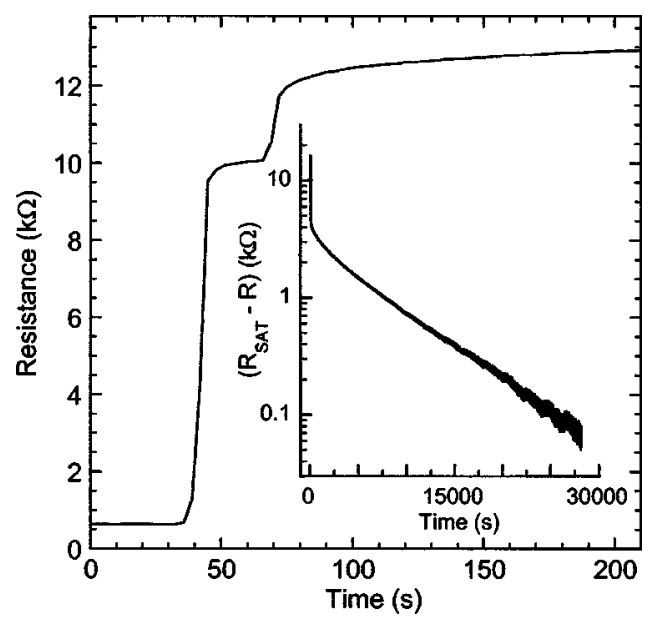

FIG. 3. Behavior of the NPPC effect before, during, and after illumination with blue light Inset shows the deviation of the resistance from its saturation value $\left(R_{\mathrm{SAT}}=17 \mathrm{k} \Omega\right)$. 


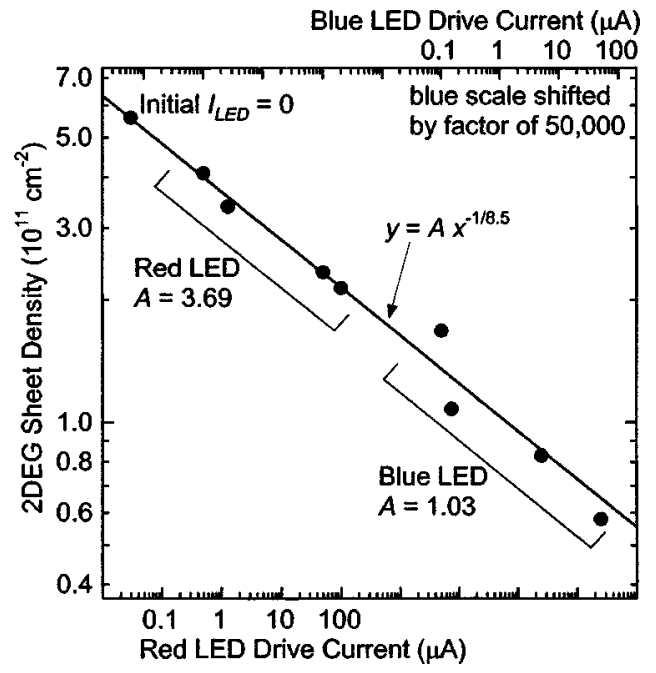

FIG. 4. Electron sheet density vs LED drive current, before (labeled initial) and after illumination with red light (labeled red LED) and blue light (labeled blue LED).

The electron sheet density as a function of LED drive current and color is shown in Fig. 4. The red LED drive current is shown on the lower $x$-axis scale and the blue LED drive current (scaled up by a factor of 50000 ) is shown on the upper $x$-axis scale. The initial density before illumination is given by the solid circle labeled initial, and the densities after illumination under red or blue light are given by the solid circles labeled red or blue LED. The straight solid line is a power law fit to the data; this fit illustrates a weak, approximately $-1 / 8$, power dependence of the sheet density on LED drive current. The 50000 scaling factor required to arrange the blue data in a line with the red data, indicates that blue light is $(50000)^{-1 / 8.5} \approx 3.6$ times more effective at reducing the 2DEG density than red light. When plotted in this manner the dependence of electron density on illumination intensity does not appear to saturate, however, due to the weakness of the power law, it appears to saturate when plotted on a linear scale. The functional form of the effect of light intensity on NPPC has not been reported, however qualitatively, a weak dependence on illumination intensity has been previously observed. ${ }^{2,7}$

For visible wavelengths, the absorption of light in the AlSb barriers and InAs QW is dominated by band-to-band absorption; therefore the photogeneration of electron-hole pairs must be the prevailing process. The absorptance (fraction of light entering layer that is absorbed) in the InAs QW is about $20 \%\left(\alpha \sim 1.5 \times 10^{5} \mathrm{~cm}^{-1}\right)$ for red $(\sim 600 \mathrm{~nm})$ light and $60 \%\left(\alpha \sim 6 \times 10^{5} \mathrm{~cm}^{-1}\right)$ for blue $(\sim 450 \mathrm{~nm})$ light. While the absorptance in the upper (40 nm thick) AlSb barrier is about $4 \%\left(\alpha \sim 1 \times 10^{4} \mathrm{~cm}^{-1}\right)$ for red light (indirect band-gap transition) and about $90 \%\left(\alpha \sim 6 \times 10^{5} \mathrm{~cm}^{-1}\right)$ for blue light (direct band-gap transition). As a result about $2 \%$ of the blue light (that enters the sample) is absorbed in the InAs QW (after passing through the $\mathrm{Ge}, \mathrm{GaSb}$, and $\mathrm{AlSb}$ layers) compared to about $13 \%$ for the red light ( $~ 7$ times less blue light is absorbed in the InAs QW). On the other hand, about $40 \%$ of the blue light is absorbed in the upper AlSb barrier compared to about $3 \%$ for red light $(\sim 13$ times more blue light is absorbed in the upper AlSb barrier). Interestingly enough, about $1 \%$ of both the red and blue light is absorbed in the lower ( $25 \mathrm{~nm}$ thick) AlSb barrier; $\sim 50 \%$ of the red light reaches the lower barrier, where little of it is absorbed, and $\sim 1 \%$ of the blue light reaches the lower barrier, where most of it is absorbed.

In conclusion, illumination with red or blue light rapidly drives the 2DEG population into a new steady state where photogenerated holes near the InAs QW drift toward, and recombine with electrons in the InAs QW. This process takes place over a few seconds and quickly terminates, indicating that the transport of holes to the InAs QW suddenly ceases; likely due to a reduction in the built-in electric field as the corresponding photogenerated electrons drift toward, and neutralize, surface donors and to a lesser extent, donors in the upper AlSb barrier. The ability of the holes to drift toward and recombine with electrons in the InAs QW weakly increases with illumination intensity and strongly increases with illumination energy; indicating that the electron-hole pair generation rate (which is proportional to intensity and several times larger for blue light), and possibly even the level excited above the band edge, play an important role in hole transport and electron-hole recombination at the InAs QW. The further rapid decrease in the 2DEG density observed once illumination is stopped is likely due to the recombination of QW electrons with some of the photogenerated hole population next to the QW. The final metastable steady state is reached after a long time relaxation of electrons that slowly move from the InAs QW to ionized deep levels in the AlSb barriers.

${ }^{1}$ C. Nguyen, B. Brar, H. Kroemer, and J. H. English, Appl. Phys. Lett. 60, 1854 (1992).

${ }^{2}$ Ch. Gauer, J. Scriba, A. Wixforth, J. P. Kotthaus, C. Nguyen, G. Tuttle, J. H. English, and H. Kroemer, Semicond. Sci. Technol. 8, S137 (1993).

${ }^{3}$ G. Tuttle, H. Kroemer, and J. H. English, J. Appl. Phys. 65, 5239 (1989).

${ }^{4}$ F.-C. Wang, W. E. Zhang, C. H. Yang, M. J. Yang, and B. R. Bennet, Appl. Phys. Lett. 69, 1417 (1996).

${ }^{5}$ I. Lo, W. C. Mitchell, M. O. Manasreh, C. E. Stutz, and K. R. Evans, Appl. Phys. Lett. 60, 751 (1992).

${ }^{6}$ W. C. Wang, L. C. Tsai, J. C. Fan, Y. F. Chen, and I. Lo, J. Appl. Phys. 86, 3152 (1999).

${ }^{7}$ P. F. Hopkins, A. J. Rimberg, R. M. Westervelt, G. Tuttle, and H. Kroemer, Appl. Phys. Lett. 58, 1428 (1991). 